\title{
Effects of nicotine stimulation on spikes, theta frequency oscillations, and spike-theta oscillation relationship in rat medial septum diagonal band Broca slices
}

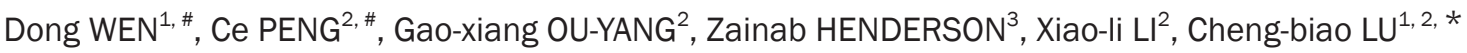 \\ ${ }^{1}$ The Key Laboratory for Computer Virtual Technology and System Integration of Hebei Province, Yanshan University, Qinhuangdao \\ 066004, China; ${ }^{2}$ School of Electrical Engineering, Yanshan University, Qinhuangdao 066004, China; ${ }^{3}$ Institute of Membrane Biology, \\ Leeds, University of Leeds, United Kingdom
}

\begin{abstract}
Aim: Spiking activities and neuronal network oscillations in the theta frequency range have been found in many cortical areas during information processing. The aim of this study is to determine whether nicotinic acetylcholine receptors (nAChRs) mediate neuronal network activity in rat medial septum diagonal band Broca (MSDB) slices.

Methods: Extracellular field potentials were recorded in the slices using an Axoprobe $1 \mathrm{~A}$ amplifier. Data analysis was performed offline. Spike sorting and local field potential (LFP) analyses were performed using Spike2 software. The role of spiking activity in the generation of LFP oscillations in the slices was determined by analyzing the phase-time relationship between the spikes and LFP oscillations. Circular statistic analysis based on the Rayleigh test was used to determine the significance of phase relationships between the spikes and LFP oscillations. The timing relationship was examined by quantifying the spike-field coherence (SFC).

Results: Application of nicotine $(250 \mathrm{nmol} / \mathrm{L})$ induced prominent LFP oscillations in the theta frequency band and both small- and large-amplitude population spiking activity in the slices. These spikes were phase-locked to theta oscillations at specific phases. The Rayleigh test showed a statistically significant relationship in phase-locking between the spikes and theta oscillations. Larger changes in the SFC were observed for large-amplitude spikes, indicating an accurate timing relationship between this type of spike and LFP oscillations. The nicotine-induced spiking activity (large-amplitude population spikes) was suppressed by the nAChR antagonist dihydro- $\beta$-erythroidine $(0.3 \mu \mathrm{mol} / \mathrm{L})$.

Conclusion: The results demonstrate that large-amplitude spikes are phase-locked to theta oscillations and have a high spike-timing accuracy, which are likely a main contributor to the theta oscillations generated in MSDB during nicotine receptor activation.
\end{abstract}

Keywords: medial septum diagonal band of Broca; theta oscillations; spike; LFP; nicotinic acetylcholine receptor; nicotine; dihydro- $\beta$ erythroidine; brain slice; electrophysiology

Acta Pharmacologica Sinica (2013) 34: 464-472; doi: 10.1038/aps.2012.180; published online 11 Mar 2013

\section{Introduction}

Local field potential (LFP) oscillations in the theta frequency band modulate neuronal activity and play important roles in memory formation, attention, arousal and recall ${ }^{[1-3]}$. Theta oscillations are present in many brain areas, including the hippocampus and cortex ${ }^{[4-8]}$. Theta oscillations control the timing of neuronal firing. Additionally, GABA (A) receptor-mediated inhibition during oscillations controls spike timing in CA1

\footnotetext{
\#These authors contributed equally to this paper.

* To whom correspondence should be addressed.

E-mail cblu@ysu.edu.cn

Received 2012-08-02 Accepted 2012-12-05
}

pyramidal neurons ${ }^{[9,10]}$. The phase relationship between the spikes and theta oscillations can be computed and used as a predictor of memory strength ${ }^{[11]}$. The relationship between spikes and LFPs is highly specific to the underlying connectivity of a local network; the LFP-spike relationship is complex and may vary within same brain area under similar task conditions $^{[2]}$.

The medial septum diagonal band Broca (MSDB), located in the basal forebrain area ${ }^{[12]}$, provides the major cholinergic and GABAergic projections to the hippocampus. It plays a pivotal role in the generation of the hippocampal theta rhythm ${ }^{[13,14]}$ and is thought to be an essential internal timing mechanism for neuronal firing in the hippocampus. In vivo, a proportion 
of septohippocampal neurons display rhythmic burst firing that is phase-locked to the hippocampal theta rhythm. Activation of the brain stem reticular formation increases hippocampal theta activity via the indirect excitation of rhythmic bursting in the MSDB ${ }^{[15]}$.

Although the MSDB sends cholinergic axons to the hippocampus ${ }^{[16]}$, it receives cholinergic input from the pedunculopontine tegmental nucleus. Cholinergic projections modulate MSDB neuronal firing at least partly through $\mathrm{nAChR}$ activation ${ }^{[17]}$. nAChR activation regulates stimulus-evoked transient gamma oscillations in the hippocampus ${ }^{[18]}$; nicotine-induced theta oscillations have also been reported ${ }^{[19]}$. It is unknown whether the tonic activation of nAChRs can induce oscillatory or spiking activity in the MSDB. The aim of this study is to determine whether $\mathrm{nAChR}$ activation can induce local network activity (ie, LFPs and spikes) and the timing of neuronal spikes relative to the theta rhythm during nicotine application.

\section{Materials and methods} Preparation of slices

All procedures were performed in accordance with the UK Animals (Scientific Procedures) Act of 1986 and associated guidelines and with the prior approval of the local ethical committee of the University of Leeds. All efforts were made to minimize animal suffering and to reduce the number of animals used. Three-week-old male Wistar rats $(n=64)$ were anesthetized by intraperitoneal injection of Sagatal (sodium pentobarbitone, $100 \mathrm{mg} / \mathrm{kg}$, Rhône Mérieux Ltd, Harlow, UK). When all pedal reflexes were abolished, the animals were perfused intracardially with chilled $\left(5^{\circ} \mathrm{C}\right)$, oxygenated artificial cerebrospinal fluid (ACSF) in which the sodium chloride had been replaced by iso-osmotic sucrose. This ACSF (305 mosmol/L) contained (in mmol/L) 225 sucrose, $3 \mathrm{KCl}, 1.25$ $\mathrm{NaH}_{2} \mathrm{PO}_{4}, 24 \mathrm{NaHCO}_{3}, 6 \mathrm{MgSO}_{4}, 0.5 \mathrm{CaCl}_{2}$, and 10 glucose. For extracellular field recording, two $450-\mu \mathrm{m}$ sagittal slices of rat brain, straddling the midline and containing the MSDB, were cut at $4-5^{\circ} \mathrm{C}$ in sucrose ACSF using a Leica VT1000S vibratome (Leica Microsystems UK, Milton Keynes, UK).

\section{Electrophysiological recording and data analysis}

For extracellular field potential recordings, two MSDB slices were transferred to an interface recording chamber. The slices were continuously perfused with ACSF using a peristaltic pump (minipuls 3, Gilson, Madison, WI, USA) at $2 \mathrm{~mL} / \mathrm{min}$. The slices were maintained at $33^{\circ} \mathrm{C}$ and kept at the interface between the ACSF and warm humidified carbogen gas (95\% $\mathrm{O}_{2}-5 \% \mathrm{CO}_{2}$ ). This ACSF contained (in mmol/L) $126 \mathrm{NaCl}, 3$ $\mathrm{KCl}, 1.25 \mathrm{NaH}_{2} \mathrm{PO}_{4}, 24 \mathrm{NaHCO}_{3}, 2 \mathrm{MgSO}_{4}, 2 \mathrm{CaCl}_{2}$ and 10 glucose. The slices were allowed to equilibrate in this medium for $1 \mathrm{~h}$ before recording.

Both channels of an Axoprobe 1A amplifier (Axon Instruments, Union City, CA, USA) were used for extracellular field recordings. Recording pipettes were glass microelectrodes containing ACSF (resistance 2-5 M 2 ). The data were bandpass filtered online between $0.5 \mathrm{~Hz}$ and $2 \mathrm{kHz}$ using the Axoprobe amplifier and a NeuroLog system NL106 AC/DC amplifier (Digitimer Ltd, Welwyn Garden City, UK). The data were digitized at a sample rate of $5-10 \mathrm{kHz}$ using a CED 1401 plus ADC board (Digitimer Ltd). Electrical interference from the mains supply was eliminated from extracellular recordings online using $50 \mathrm{~Hz}$ noise eliminators (HumBug; Digitimer Ltd).

The data were analyzed off-line using software from Spike2 (CED, Cambridge, UK) and MATLAB (MathWorks, Natick, Massachusetts, USA). Power spectra were generated to provide a quantitative measure of the frequency components in a stretch of recording. Power, a quantitative measure of the oscillation strength, was plotted against the respective frequency. Power spectra were constructed for 30-60 s epochs of extracellular field recordings using a fast Fourier transform algorithm provided by MATLAB. The parameters used for measuring the oscillatory activity in the slice were peak frequency $(\mathrm{Hz})$ and area power $\left(\mu \mathrm{V}^{2}\right)$. In this study, area power was equivalent to the computed area under the power spectrum between the frequencies of 4 and $12 \mathrm{~Hz}$. Spikes were detected and counted by the Spike2 software if the spike was more than one-third of the average spike height during the field population spiking activity.

Spike sorting was performed off-line using the Spike2 software; spikes were matched with templates generated by Spike2 based on the spike waveform shape.

\section{Estimation of phase-locking and spike-field coherence}

LFPs were recorded from the same electrode as the spiking activity. To investigate the strength of theta frequency phaselocking, the LFPs were band-pass filtered in the theta band (4-12 Hz) using a two-way least-squares FIR filter (the eegfilt. $m$ routine from the EEGLAB toolbox). The phase was estimated using the Hilbert transform (HT), a well-known transformation that is used to determine the instantaneous phase of a real signal $x(t)$. Essentially, the HT is used to compute a complex signal $Y(t)$, called the analytical signal corresponding to $x(t)$, defined by

$$
Y(t)=x(t)+j \cdot h(t)
$$

where $h(t)$ is the HT of $x(t)$. If $x(t)$ is band-pass filtered for the theta frequency, then

$$
\phi(t)=\tan ^{-1}\left[\frac{h(t)}{x(t)}\right]
$$

where $\phi(t)$ is the phase function of the complex signal $Y(t)$ corresponding to $x(t)$. The phase of each spike at the theta frequency was then determined using the results of the HT transform. The phase was measured (in radians) in the range $-\Pi / 2$ to $+\Pi / 2$ and was reflected to the range 0 to $2 \Pi\left(0^{\circ}\right.$ to $\left.360^{\circ}\right)$ with the phases zero and $2 \Pi$ equal to the peak, and phase $\Pi$ equal to the trough of the oscillation. The sample of all phase angles $\Phi_{\mathrm{i}}$ $(i=1, \ldots, n)$ defines the first trigonometric moment

$$
m^{\prime}=(1 / n) \sum_{i=1}^{n} e^{j \phi_{\mathrm{i}}}=\bar{R} e^{j \mu}
$$

The mean direction or preferred phase $\mu$ of the sample is given by the orientation of $m^{\prime}$, and the mean resultant length $\bar{R}$ is given by the modulus of $m^{\prime}$.

The Rayleigh test determines the significance of the mean 
phase direction in a cycle histogram. The spikes had accurate phase-locking performance for low $P$ values. The Rayleigh statistic is $Z=n \bar{R}^{2}$, and the probability that the null hypothesis of sample uniformity holds is given by

$P=e^{-Z}\left[1+\left(2 Z-Z^{2}\right) /(4 n)-\left(24 Z-132 Z^{2}+76 Z^{3}-9 Z^{4}\right) /\left(288 n^{2}\right)\right]$

To guarantee sufficient statistical power, only neurons that fired at least 50 spikes were evaluated for phase-locking.

To analyze the data further, the spike-field coherence (SFC) was quantified to examine the timing relationship between the spikes of single neurons and the ongoing theta oscillations. The SFC is the ratio of the frequency spectrum of the spiketriggered average (STA) to the average spectrum of the LFP traces (spike-triggered power or STP). The SFC is a function of frequency $f$ and ranges from $0 \%$ to $100 \%$. Formally, the $\operatorname{SFC}(f)=[\operatorname{STA}(f) / \operatorname{STP}(f)] \times 100 \%$.

For every spike, the STA was constructed by extracting a 200 ms time window of the LFP, centered on the spike. Averaging these traces of LFPs produced the STA. STP quantifies the power of the oscillations that are present in LFPs and is calculated by averaging the spectra of individual LFP traces.

All statistical tests were performed using SigmaStat software (SPSS Inc, CA, USA). The results are expressed as the mean \pm standard error of the mean. The significance of comparisons between two groups was determined using Student's $t$ test or rank-sum test. Comparisons of more than two groups were made using either one-way analysis of variance (ANOVA) on the ranks or repeated-measures ANOVA. For all measures in this study, $P<0.05$ was considered significant.

\section{Drugs used for electrophysiology}

Except where indicated, all standard reagents were obtained from either Sigma-Aldrich (UK) or VWR International (Lutterworth, UK). Nicotine and dihydro- $\beta$-erythroidine (DH $\beta E$ $0.3 \mu \mathrm{mol} / \mathrm{L}$ ) were purchased from Tocris Cookson, Ltd (Bristol, UK). Stock solutions at $10^{3}$ of the working concentration were made in water and stored in individual aliquots at $-45^{\circ} \mathrm{C}$. Working solutions were freshly prepared on the day of the experiment. The drugs were applied by bath perfusion.

\section{Results}

LFPs were recorded from MSDB slices using extracellular electrodes. In a proportion of MSDB slices $(n=6)$, no spikes or persistent oscillatory activity was detected in control conditions. Nicotine ( $250 \mathrm{nmol} / \mathrm{L})$ induced only small-amplitude population spikes $(\leq 50 \mu \mathrm{V})$ and oscillatory activity at the theta frequency band (theta oscillations) (Figure 1A, 1C). The spikes (in blue) and oscillatory activity (in red) were extracted using either high-pass $(300 \mathrm{~Hz})$ or band-pass filtering $(4-12 \mathrm{~Hz}$ ) (Figure 1B, 1D). The waveform of the sorted spikes is presented in Figure 1E. The interspike intervals for all spikes were longer than or equal to $3 \mathrm{~ms}$ (Figure 1F), validating that these spikes were biological signals. A histogram of spike phases indicated that the mean phase $\left(165^{\circ}\right)$ of spikes was near the trough of the theta oscillations (Figure 1G).

The STA of the LFP was used to measure local neuronal synchronization. The STA was constructed by averaging LFP seg- ments of $200 \mathrm{~ms}$ centered on every spike. As shown in Figure $1 \mathrm{H}$ and $1 \mathrm{I}$, the flat STA traces and very small power spectrum of the STA indicated that the spikes had no temporal relationship to the LFP oscillations. In Figure 1J, the average spectrum of all LFP segments was shown, and it was used to construct the STA (STP).

The SFC was also used to examine the timing relationship between the spikes of single neurons and the ongoing theta oscillations. A larger SFC indicated that the spikes more accurately followed a particular phase of this frequency. The SFC is the ratio of the frequency spectrum of STA to STP. As expected, the SFC was not significant (Figure 1K).

In a set of MSDB slices $(n=9)$, small-amplitude population spikes $(\leq 50 \mu \mathrm{V})$ and LFPs were detected in the control condition. Nicotine application induced relatively large-amplitude population spikes (50-99 $\mu \mathrm{V}$ ) and the LFP (Figure 2A, 2C).

In the example shown in Figure $2 \mathrm{~A}, 2 \mathrm{C}, 2 \mathrm{E}$, and $2 \mathrm{H}$, nicotine increased the number of small-amplitude population spikes from $1.1 \mathrm{~Hz}$ to $6.7 \mathrm{~Hz}$ and induced large-amplitude population spikes $(3.4 \mathrm{~Hz})$. In this example experiment, we observed low-frequency $(<10 \mathrm{~Hz})$ oscillations that were present before the application of nicotine. Nicotine increased the oscillatory frequency (from 7 to $10 \mathrm{~Hz}$ ) and the power (Figure 2B, 2D).

The STA traces showed a deviation from zero, indicating a relationship between spike timing and the LFP. In our experiments, the STA trace deviation, fSTA, and percentage change in the SFC for small-amplitude spikes in both the control condition and in the presence of nicotine were relatively small (Figure 2N, 2O, 2Q-2S, 2U, and Figure 2G, 2J). The STA trace deviation, $\mathrm{SSTA}$, and percentage change in the SFC were significantly higher for the relatively large-amplitude spikes (Figure $2 \mathrm{~V}, 2 \mathrm{~W}, 2 \mathrm{Y}$ ) in the nicotine group than in the control. The STA of phase-locked neurons showed strong oscillations in the $8-12 \mathrm{~Hz}$ range (Figure 2O, 2S, 2W). These results suggest that neurons with $\mathrm{nAChR}$ activation can generate relatively large spikes that are synchronized better during oscillatory activity.

In another set of MSDB slices $(n=7)$, small-amplitude population spikes and small but significant theta oscillations were detected in the control condition; after nicotine application, significant amounts of large-amplitude populations spikes $(\geq 100 \mu \mathrm{V})$ were recorded. In the example shown in Figure $3 \mathrm{~A}, 3 \mathrm{C}, 3 \mathrm{E}, 3 \mathrm{H}$, and $3 \mathrm{~K}$, nicotine increased the frequency of small-amplitude population spikes from $1.1 \mathrm{~Hz}$ to $3.7 \mathrm{~Hz}$ and induced large-amplitude population spikes $(3.4 \mathrm{~Hz})$. In this example experiment, prominent low-frequency $(<10 \mathrm{~Hz})$ oscillations were present before the application of nicotine, and nicotine increased the power of the theta oscillations (Figure 3B, 3D).

Neurons had a range of phase preferences (Figure 3G, 3J, $3 \mathrm{M})$, but the mean phases of these spikes relative to the theta oscillations differed. For the small-amplitude population spikes in control conditions, the mean phase compared to the theta oscillation was $175^{\circ}$. After nicotine application, the mean phase of the large- and small-amplitude population spikes relative to the theta oscillation were near the initial rising phase $\left(210^{\circ}\right)$ and the trough $\left(165^{\circ}\right)$ of the LFP, respectively. Interest- 

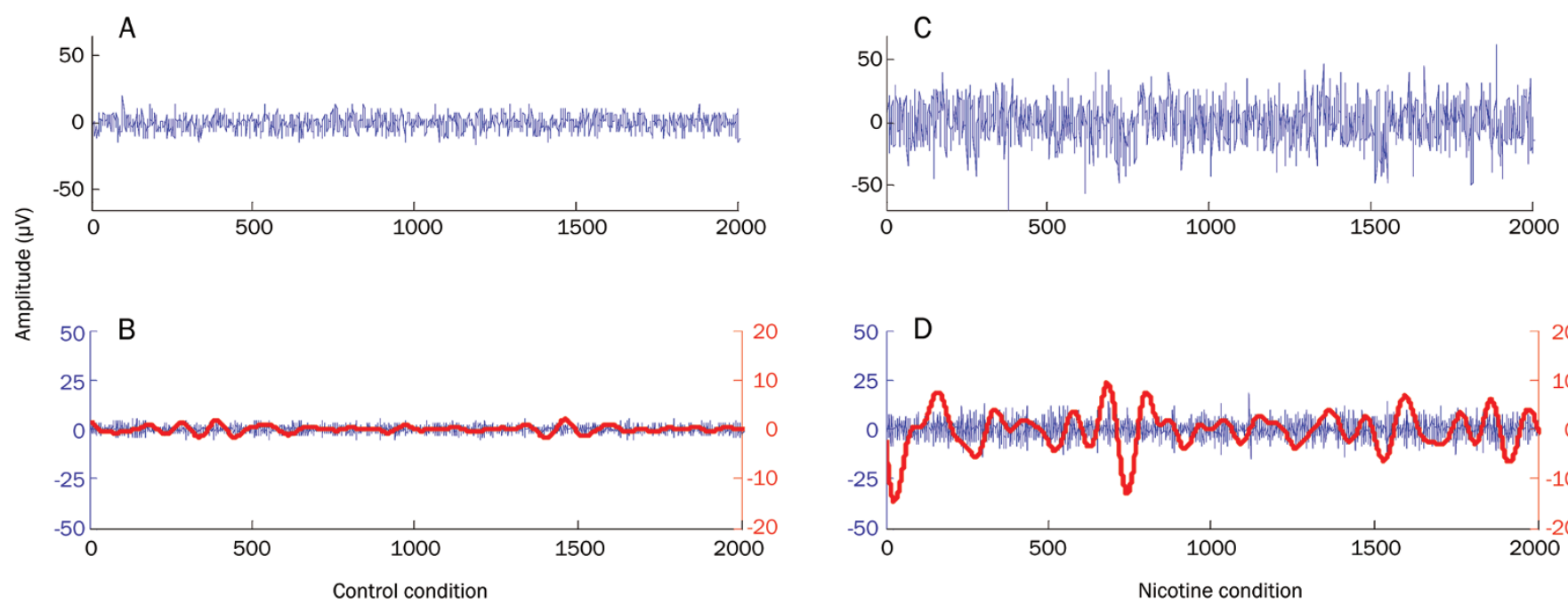

E

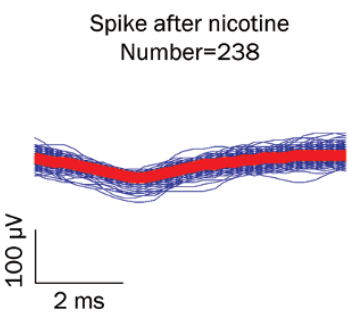

$\mathrm{F}$

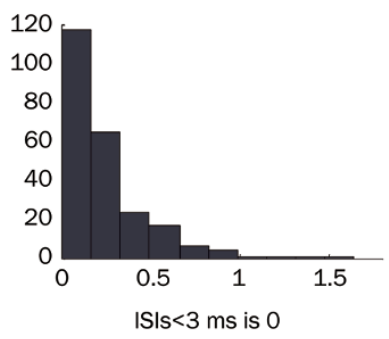

G

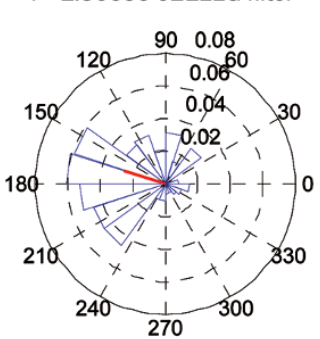

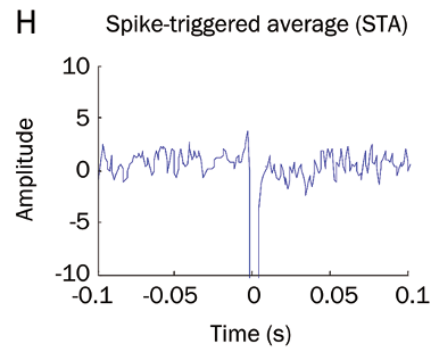
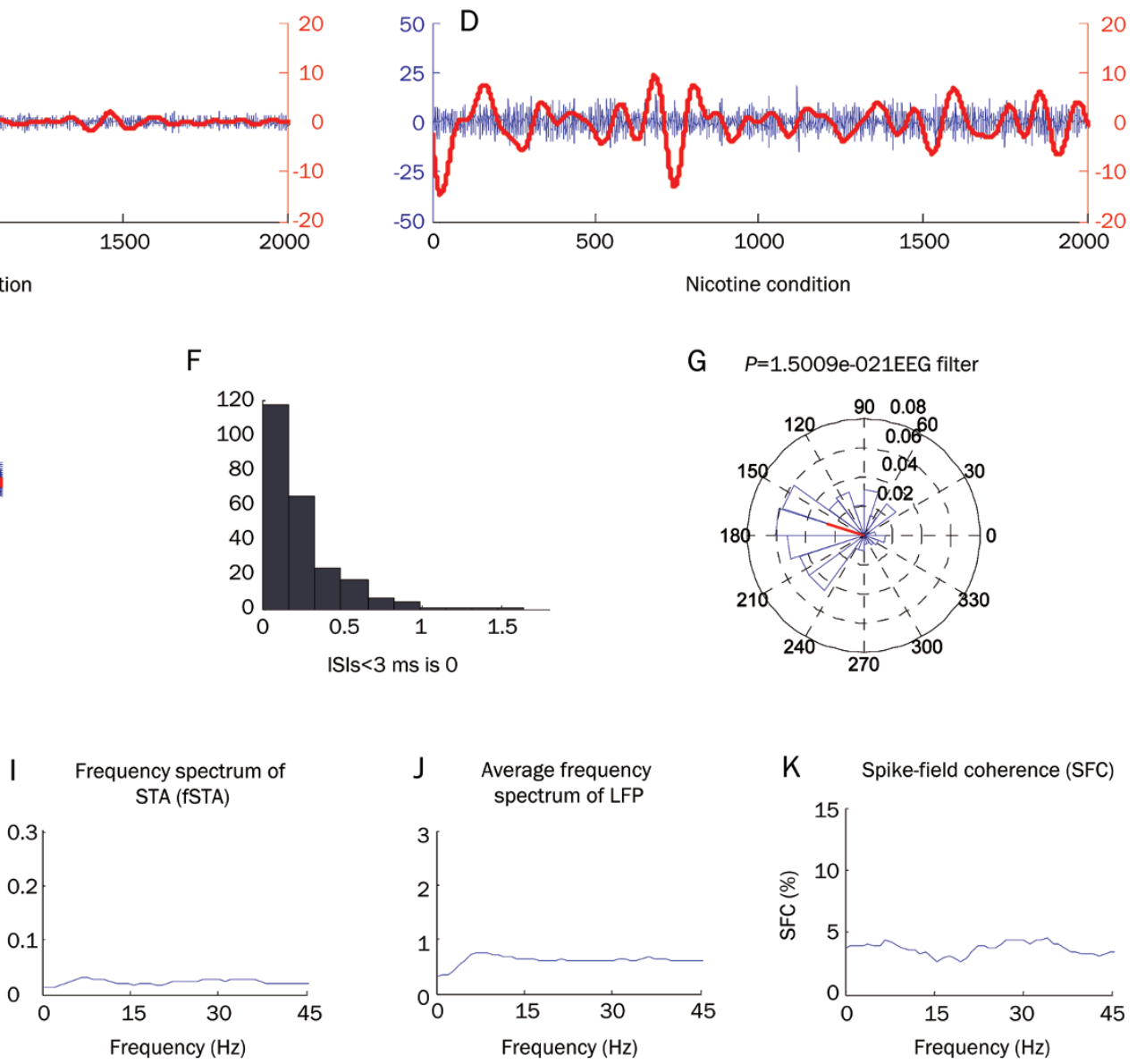

20

10

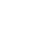




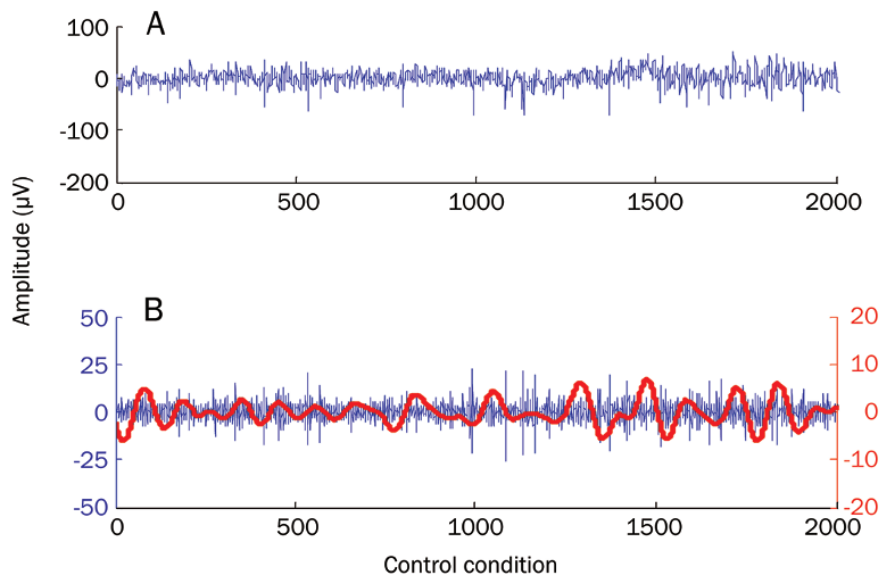

E

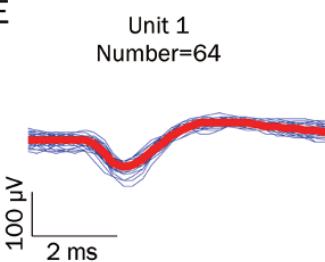

$\mathrm{H}$

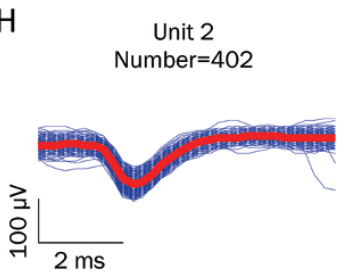

K

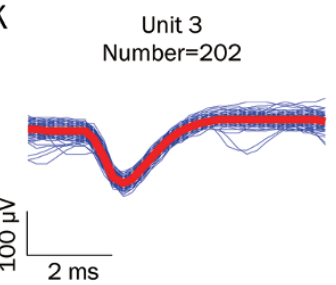

$\mathrm{F}_{30}$

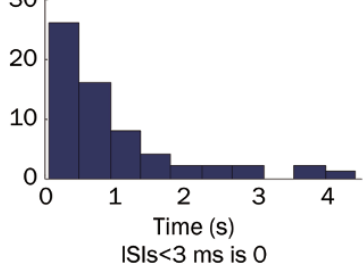

Spike before nicotine

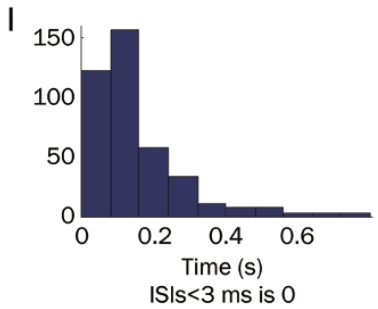

L

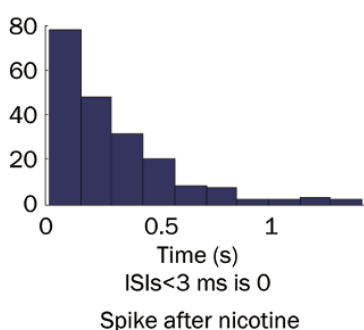

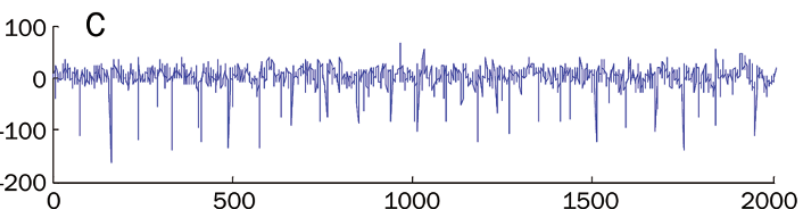

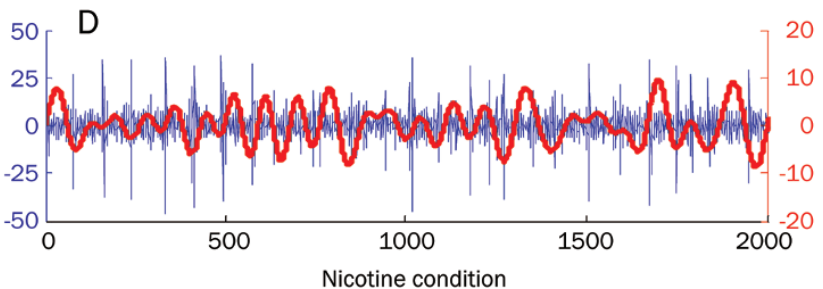

G $P=0.00028012$ EEG filter

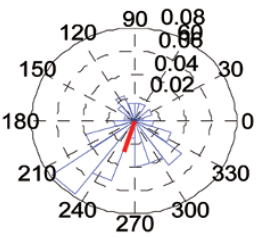

J $P=3.3716 \mathrm{e}-017 \mathrm{EEG}$ filter

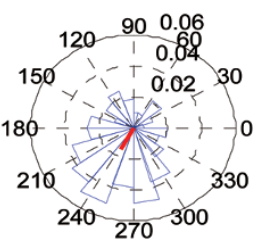

M $P=6.2435 \mathrm{e}-033$ EEG filter

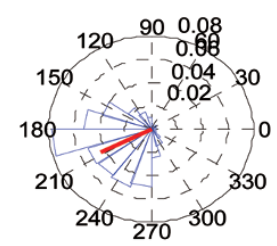

Figure 2-1. Nicotine-induced intermediate amplitude spikes (51-99 $\mu \mathrm{V})$ and theta oscillatory activities. (A, B) Low-level spontaneous neuronal activity in the control condition. (C, D) Spikes and LFPs in the presence of nicotine. The sampling rate was $1000 \mathrm{~Hz}$. ((B, D) Spikes (300 Hz high-pass filtered, blue line) and the low-frequency component of the LFP (pass band 4-12 Hz, red line). (E, H, K) Waveforms of sorted spikes indicate multiple unit recording before $(E)$ and after nicotine application $(H, K)$. ( $F, I, L)$ Interspike interval frequency plot. (G, J, M) Histograms of spike phases of three waveforms for the $4-12 \mathrm{~Hz}$ oscillations. (G) The mean phase $\left(250^{\circ}\right)$ is in the initial rising phase toward the peak $360^{\circ}(P=0.0002801)$ in the control condition. (J, M) Histograms of spike phases for small and relatively large population spikes for the $4-12 \mathrm{~Hz}$ oscillations were at $240^{\circ}(P<10-17$, Rayleigh test) and $210^{\circ}(P<10-33$, Rayleigh test $)$ in the presence of nicotine. The mean phases are near trough, but in the initial rising phase.

strong, accurate timing relationship between the large-amplitude population spikes and LFP oscillations, as measured by large percentage changes in the SFC. The LFP-spike relationship can predict memory strength ${ }^{[3,20]}$; the strong phase and timing relationship between nicotine-induced large-amplitude spikes and theta oscillations suggests that nicotine receptor activation plays a powerful role in memory enhancement. Our results also suggest that combining methods to assess phaselocking and SFC can provide a better description of spike-LFP oscillations.

Nicotine can induce both small- and large-amplitude population spikes. The mean phase of small-amplitude population 
N Spike-triggered average (STA)

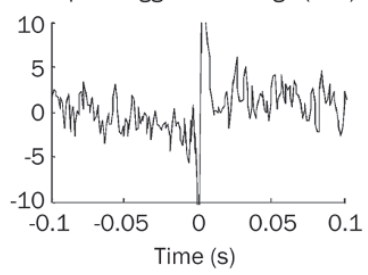

R Spike-triggered average (STA)

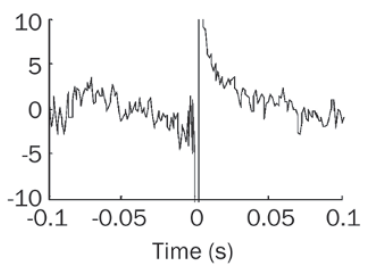

$\mathrm{V}$ Spike-triggered average (STA)

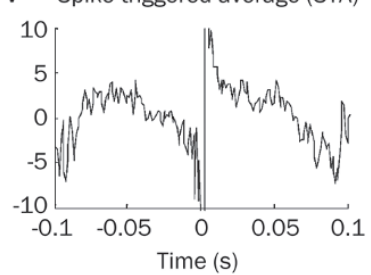

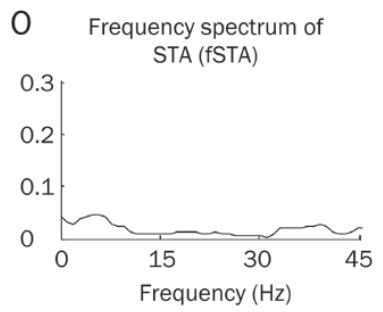

P Average frequency spectrum

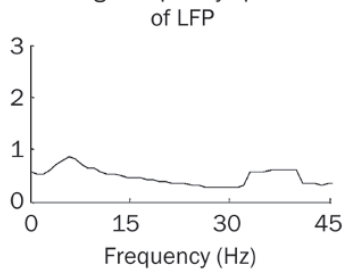

Unit 1 spike SFC before nicotine

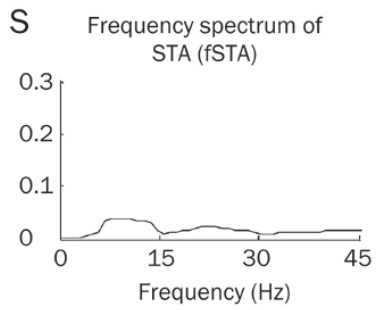

$\mathrm{T}$ Average frequency spectrum

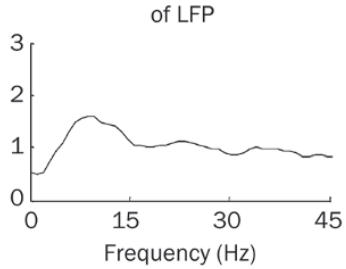

U Spike-field coherence

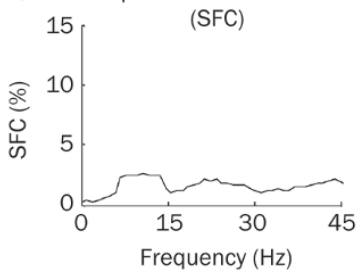

Unit 2 spike SFC after nicotine
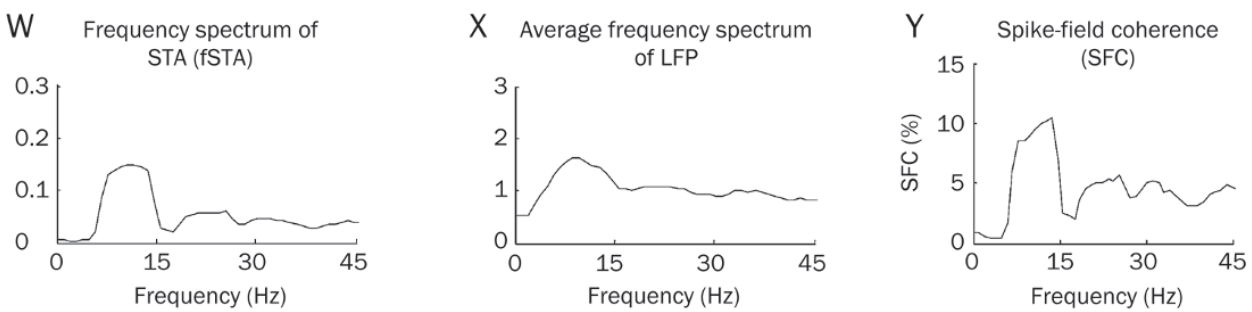

Unit 3 spike SFC after nicotine

Figure 2-2. (N) Spike-triggered average (STA) of spikes within $60 \mathrm{~s}$. (O) Frequency spectrum of STA. (P) The spike-triggered power (STP) is the average spectrum of all LFP segments used to construct the STA. (Q, U, Y) Spike-field coherence (SFC) before (Q) and after nicotine (U, Y). SFC was calculated as the ratio of the spectrum of STA divided by STP. The threshold for the spike sorting was $\pm 50 \mu \mathrm{V}$.

spikes is $165^{\circ}$, located in the falling phase near the trough $\left(180^{\circ}\right)$ of the LFP. The mean phase of large-amplitude population spikes is $210^{\circ}$, and these are located in the initial rising phase. The difference in phase-locking to the theta oscillation between small- and large-amplitude spikes suggests that these spikes belong to different types of neurons rather than the same type of neurons in different locations. The MSDB has been reported to contain three different types of neurons with distinct firing properties: fast firing, slow firing, and burst firing $^{[21]}$.

The STA is very sensitive for detecting neuronal synchronization because the LFP averages over many spikes. If spike times have a reliable temporal relationship to the local neuronal activity (ie, the LFP), then LFP fluctuations add up during the spike-triggered averaging process. Conversely, if spike times have no temporal relationship to the activity of the surrounding neurons, fluctuations in the LFP average out during STA compilation, thus producing a flat STA. A flat STA was observed in the first case of this study, although the phase-locking of these spikes to LFP oscillations appeared to be significant (Rayleigh test). This suggests that the phase relationship alone is not enough to describe the spike-LFP relationship. In the second and third cases, strong phase-locking to the theta oscillations and prominent STA fluctuations were observed for large-amplitude spikes during nicotine stimulation. This is consistent with a previous observation that nicotine increases the power of theta oscillations and the number of population spikes (data not shown). These data suggest that nicotine receptor activation modifies the spike-timing accuracy in the MSDB. Previous studies demonstrated that the spike-timing accuracy was apparent in both the amygdala and hippocampus, suggesting that both structures can regulate memory strength ${ }^{[3]}$. We now propose that the MSDB also contains neurons that are phase-locked to theta oscillations, supporting a role of the MSDB in memory.

The STA results showed that nicotine induced neuronal oscillations at both low $(3-8 \mathrm{~Hz})$ and high $(8-12 \mathrm{~Hz})$ theta frequencies. This may reflect the complicated structures and neuronal arrangement in the MSDB or difficulty in finding the exact structures for electrode placement ${ }^{[22]}$.

Previous studies have reported that both excitatory and inhibitory neurotransmission contribute to the theta oscillations in the $\operatorname{MSDB}^{[19,23]}$. It is not clear whether the smalland large-amplitude spikes correlate with the two different types of neurons mentioned above ${ }^{[22]}$. Given that these spikes are phase-locked differently to the theta oscillations, further 

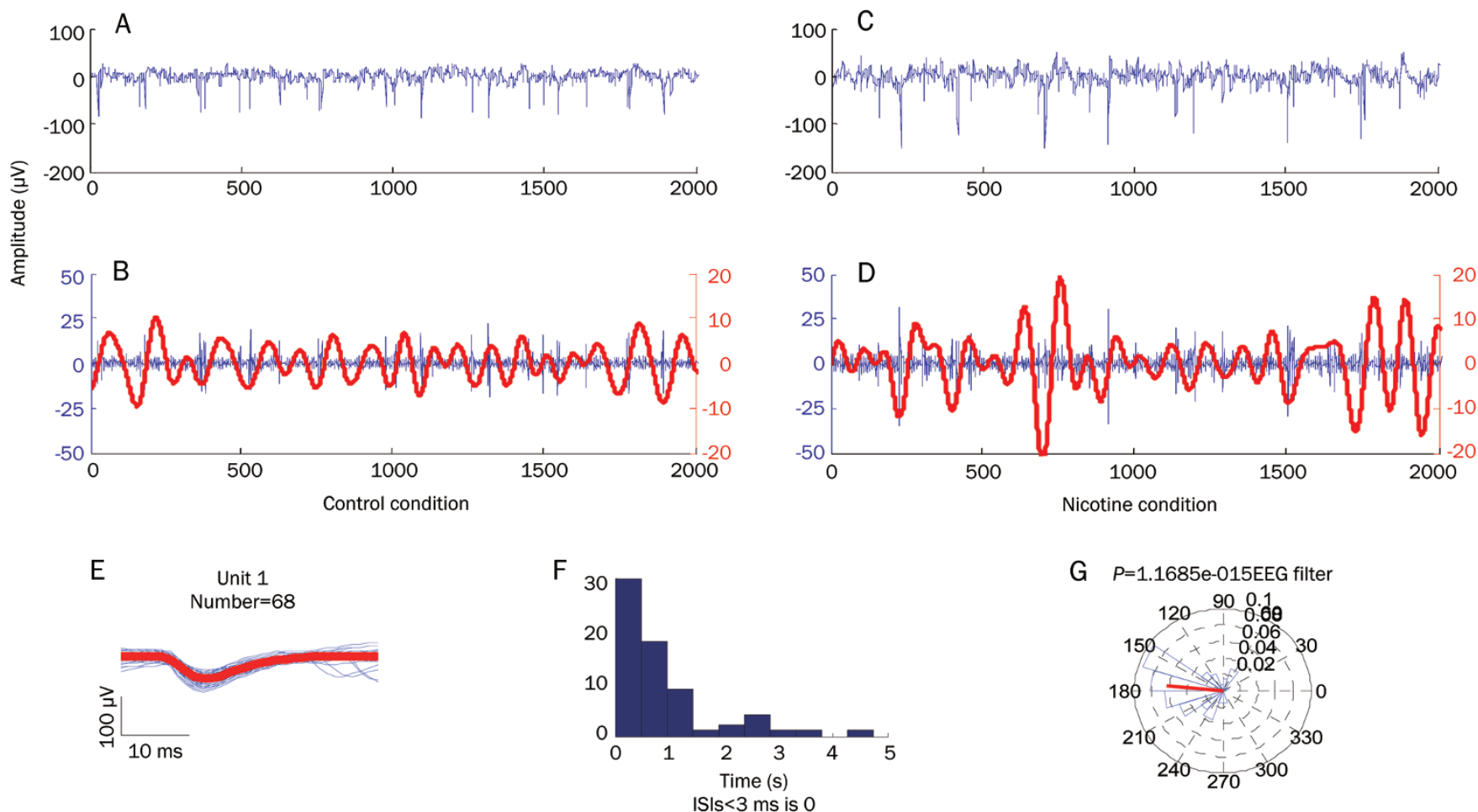

Spike before nicotine
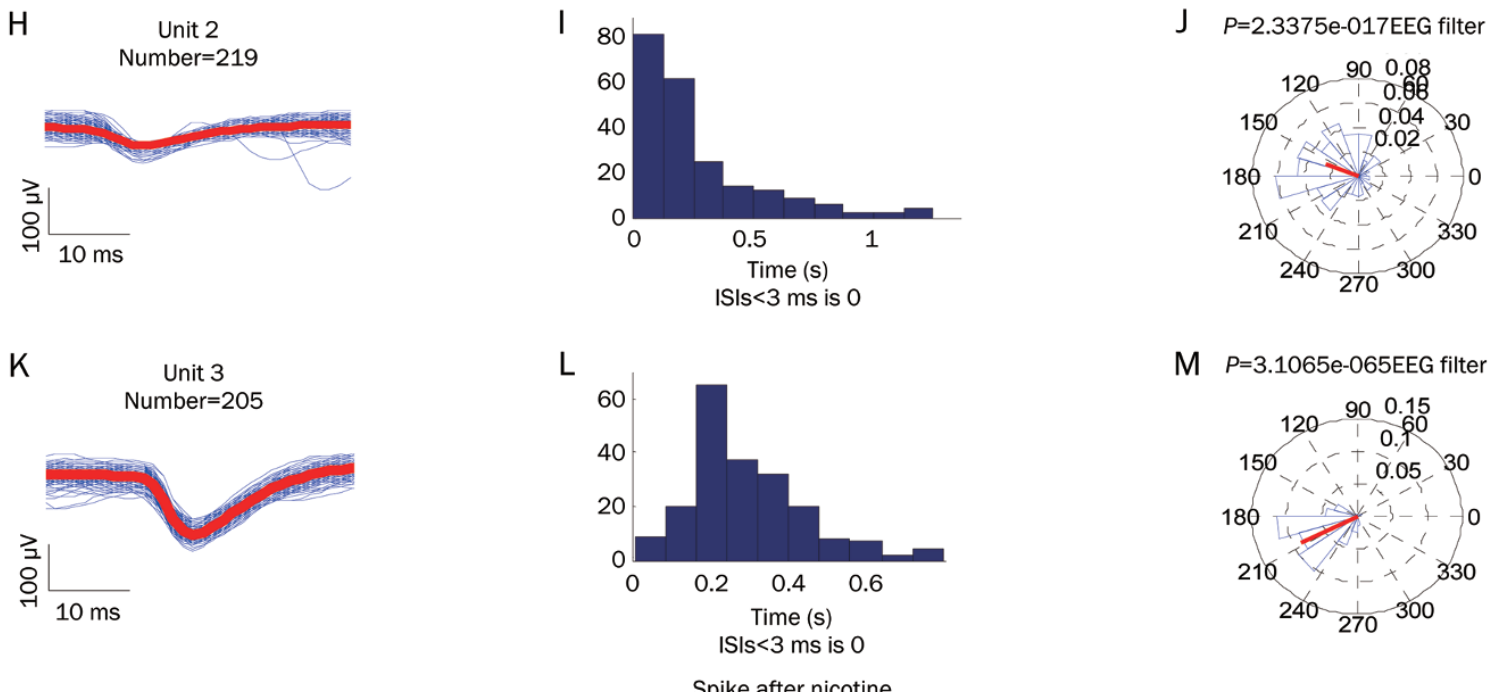

M $P=3.1065$ e-065EEG filter

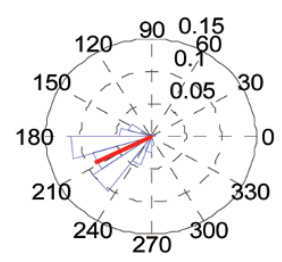

Figure 3-1. Nicotine-induced large amplitude spikes $(\geq 100 \mu \mathrm{V})$ and theta oscillatory activities. The sampling rate is $1000 \mathrm{~Hz}$. (B, D) Spikes (300 $\mathrm{Hz}$ high-pass filtered, blue line) and the low-frequency component of the LFP (pass band 4-12 Hz, red line). (E, F, G) Waveforms from neurons of the single type before nicotine application. (F) Interspike interval frequency plot. (G) Histogram of small-amplitude population spike phase for the $4-12 \mathrm{~Hz}$ oscillations. The mean phase $\left(175^{\circ}\right)$ is at the rising edge toward the peak at $360^{\circ}(P=0.032037)$. (H-M) Waveforms from neurons of different types after nicotine application. (J) Histogram of middle-amplitude population spike phase $\left(165^{\circ}\right)$ for the $4-12 \mathrm{~Hz}$ oscillations $(\mathrm{P}<10-17$, Rayleigh test). (M) Histogram of large-amplitude population spikes for the $4-12 \mathrm{~Hz}$ oscillations. The mean phase $\left(206^{\circ}\right)$ is also the start of the rising phase $(P<10-65$, Rayleigh test).

research using intracellular recording and immunocytochemistry is warranted to identify the types of these spiking neurons.

\section{Acknowledgements}

This work was supported by the National Natural Science Foundation of China (grant № 31070938 and № 81271422), the open project of the State Key Laboratory of Cognitive 


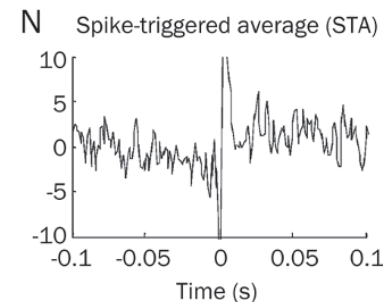

R Spike-triggered average (STA)

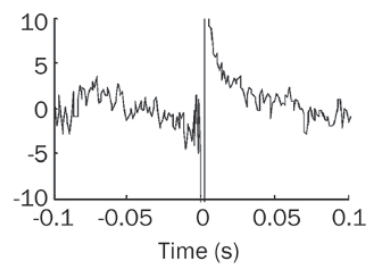

$\mathrm{V}$ Spike-triggered average (STA)

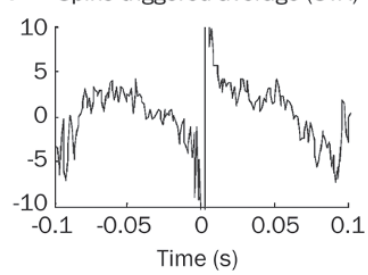

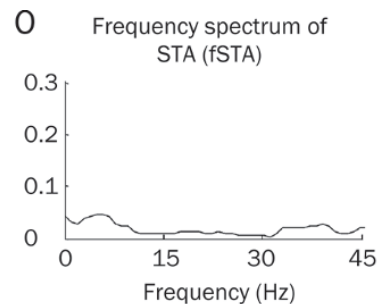

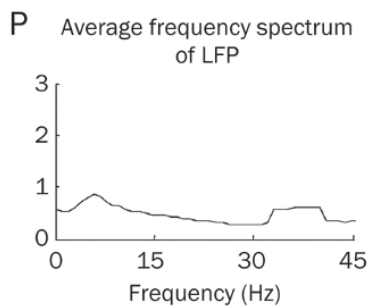

SFC before nicotine
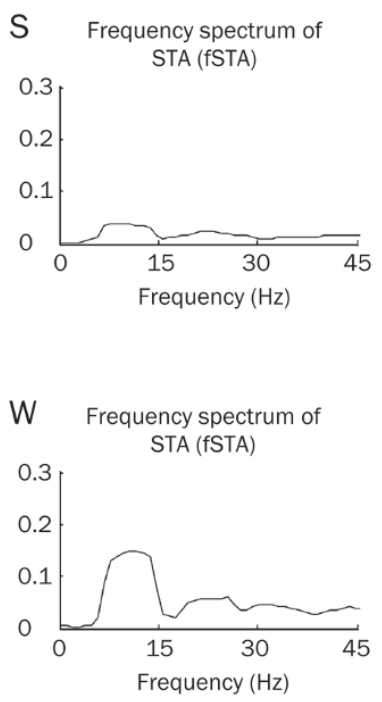
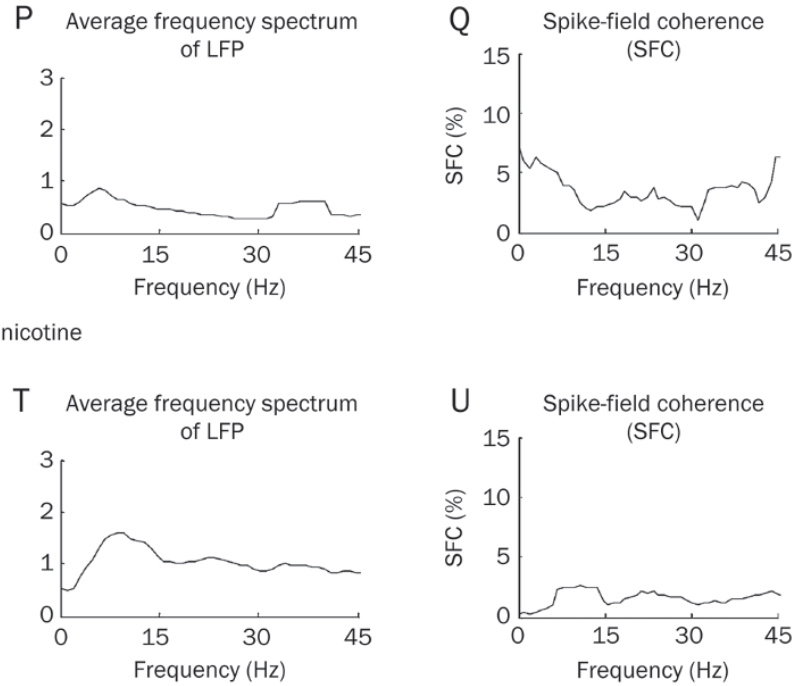

$\mathrm{X}$ Average frequency spectrum

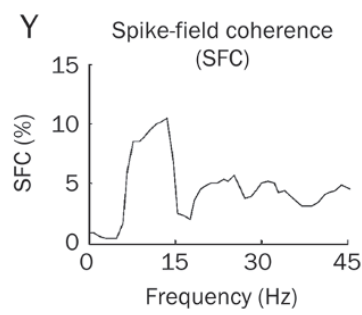

SFC after nicotine

Figure 3-2. (N-Q) Spike SFC before nicotine application. (N) Spike-triggered average (STA) of spikes within 60 s. (O) Frequency spectrum of STA. (P) The spike-triggered power (STP) is the average spectrum of all LFP segments used to construct the STA. (Q) Calculation of spike-field coherence (SFC), a ratio of the spectrum of STA divided by STP. (R-Y) SFC spikes after nicotine application. The boundaries of the thresholds in spike sorting are $\pm 50 \mu \mathrm{V}$.

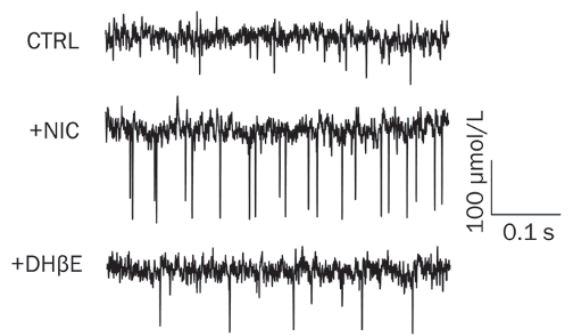

Figure 4. Nicotine induced spiking activities are reduced by nicotinic acetylcholine receptor blocker DHßE. Example traces show spiking activities induced by NIC in the presence and absence of DHßE. Traces are $1 \mathrm{~s}$ recordings.

Neuroscience and Learning in Beijing Normal University (time periods: form 2012 to 2013), Hebei applied basic research plan key program for basic research of China (grant No 12966119D), Natural Science Foundation of Hebei Province of China (grant № H2012203067), Specialized Research Fund for the Doctoral Program of Higher Education of China (grant № 20101333110006), Hebei Province Department of Education Fund (grant № 2010285) and UK Medical Research Council (grant № G0500823).

\section{Author contribution}

Dong WEN analyzed data and wrote paper; Ce PENG analyzed data and wrote paper; Zainab HENDERSON designed research; Xiao-li LI analyzed data; Cheng-biao LU designed and performed experiments and wrote paper.

\section{References}

1 Berry SD, Thompson RF. Prediction of learning rate from the hippocampal electroencephalogram. Science 1978; 200: 1298-300.

2 Hwang EJ, Andersen RA. Effects of visual stimulation on LFPs, spikes, and LFP-spike relations in PRR. J Neurophysiol 2011; 105: 1850-60.

3 Rutishauser U, Ross IB, Mamelak AN, Schuman EM. Human memory strength is predicted by theta-frequency phase-locking of single neurons. Nature 2010; 464: 903-7.

4 Buzsaki G. Theta oscillations in the hippocampus. Neuron 2002; 33: 325-40.

5 Cantero JL, Atienza M, Stickgold R, Kahana MJ, Madsen JR, Kocsis B. Sleep-dependent theta oscillations in the human hippocampus and neocortex. J Neurosci 2003; 23: 10897-903.

6 Kahana MJ, Sekuler R, Caplan JB, Kirschen M, Madsen JR. Human theta oscillations exhibit task dependence during virtual maze navigation. Nature 1999; 399: 781-4.

7 Lee H, Simpson GV, Logothetis NK, Rainer G. Phase locking of single neuron activity to theta oscillations during working memory in monkey extrastriate visual cortex. Neuron 2005; 45: 147-56. 
8 Pare D, Gaudreau H. Projection cells and interneurons of the lateral and basolateral amygdala: distinct firing patterns and differential relation to theta and delta rhythms in conscious cats. J Neurosci 1996; 16: 3334-50.

9 Jacobs J, Kahana MJ, Ekstrom AD, Fried I. Brain oscillations control timing of single-neuron activity in humans. J Neurosci 2007; 27: 3839-44.

10 Kwag J, Paulsen 0. Bidirectional control of spike timing by GABA(A) receptor-mediated inhibition during theta oscillation in CA1 pyramidal neurons. Neuroreport 2009; 20: 1209-13.

11 Anderson S. Theta phase-locked spike timing in the hippocampus: predicting strong memories. Neurosurgery 2010; 67: N17-19.

12 Leranth C, Vertes RP. Median raphe serotonergic innervation of medial septum/diagonal band of broca (MSDB) parvalbumin-containing neurons: possible involvement of the MSDB in the desynchronization of the hippocampal EEG. J Comp Neurol 1999; 410: 586-98.

13 Borhegyi Z, Varga V, Szilagyi N, Fabo D, Freund TF. Phase segregation of medial septal GABAergic neurons during hippocampal theta activity. J Neurosci 2004; 24: 8470-9.

14 Lu CB, Ouyang G, Henderson Z, Li X. Induction of theta-frequency oscillations in the rat medial septal diagonal band slice by metabotropic glutamate receptor agonists. Neuroscience 2011; 177: 1-11.

$15 \mathrm{Klemm}$ WR. Effects of electric stimulation of brain stem reticular formation on hippocampal theta rhythm and muscle activity in unanesthetized, cervical- and midbrain-transected rats. Brain Res 1972; 41: 331-44.
16 Oh JD, Butcher LL, Woolf NJ. Thyroid hormone modulates the development of cholinergic terminal fields in the rat forebrain: relation to nerve growth factor receptor. Brain Res Dev Brain Res 1991; 59: 133-42.

17 Wu J, George AA, Schroeder KM, Xu L, Marxer-Miller S, Lucero L, et al. Electrophysiological, pharmacological, and molecular evidence for alpha7-nicotinic acetylcholine receptors in rat midbrain dopamine neurons. J Pharmacol Exp Ther 2004; 311: 80-91.

18 Song C, Murray TA, Kimura R, Wakui M, Ellsworth K, Javedan SP, et al. Role of alpha7-nicotinic acetylcholine receptors in tetanic stimulationinduced gamma oscillations in rat hippocampal slices. Neuropharmacology 2005; 48: 869-80

19 Lu CB, Henderson Z. Nicotine induction of theta frequency oscillations in rodent hippocampus in vitro. Neuroscience 2010; 166: 84-93.

20 Shrager Y, Kirwan CB, Squire LR. Activity in both hippocampus and perirhinal cortex predicts the memory strength of subsequently remembered information. Neuron 2008; 59: 547-53.

21 Lu CB, Yanagawa Y, Henderson Z. Properties of gamma frequency oscillatory activity induced in hippocampal slices from the adult GAD67-GFP (Deltaneo) mouse. Brain Res 2010; 1323: 65-73.

22 Garner HL, Whittington MA, Henderson Z. Induction by kainate of theta frequency rhythmic activity in the rat medial septum-diagonal band complex in vitro. J Physiol 2005; 564: 83-102.

23 Carretie L, Hinojosa JA, Albert J, Mercado F. Neural response to sustained affective visual stimulation using an indirect task. Exp Brain Res 2006; 174: 630-7. 